\title{
Subclinical Systolic Myocardial Dysfunction in Diabetic Patients by 2-Dimensional Speckle Tracking
}

Muhammad Adel Attia, Muhammad Saad Reihan, Abu Bakr Muhammad Bakr*

Department of Cardiology, Faculty of Medicine, Al-Azhar University, Damietta, Egypt

* Corresponding author: Abu Bakr Muhammad Bakr, Mobile: (+20)01016069202, E-mail: tantawy_wael@yahoo.com

\begin{abstract}
Background: Diabetic cardiomyopathy became a subject of great interest and the evidence for this entity is rapidly emerging as the incidence of heart failure is increasing in those patients even with no hypertension or coronary artery disease (CAD). Objective: The current study was aimed at early detection of subtle left ventricular systolic dysfunction by speckle tracking echocardiography in patients with Diabetes Mellitus with and without hypertension. Patients and methods: This study included a total of 100 asymptomatic poorly controlled diabetic patients with or without hypertension and no structural heart disease and 25 age-matched apparently healthy control subjects attending at Cardiology department, Al-Azhar University Hospital in Damietta. The included subjects were divided into three groups; Group I (control) consisted of 25 apparently healthy subjects, Group II (diabetic normotensive group) consisted of 64 patients and Group III (diabetic hypertensive) consisted of 36 patients. The following were done for all: resting ECG, HbA1c, conventional echo assessment and finally strain imaging with measurement of LV-GLS and LV-GCS. Results: There was a significant inverse correlation between the duration diabetes, BMI and LV-GLS. On the other hand, there was no significant correlation between hypertension or HbA1c and echocardiographic parameters including conventional and strain methods. Patients with higher BMI had more decrease in LV circumferential strain while patients with longer periods of DM and higher values of BMI had a decrease on both global LV longitudinal and circumferential strains.
\end{abstract}

Conclusion: It could be concluded that assessment of left ventricular (LV) systolic function in patients with diabetes mellitus by speckle tracking echocardiographic modalities can detect subtle LV dysfunction earlier than conventional echocardiographic measures.

Keywords: DM, CAD, LV.

\section{INTRODUCTION}

The incidence and mortality of diabetes mellitus (DM) in and of itself may be increased in patients with congestive heart failure, irrespective of their hypertensive or ischemic heart disease status ${ }^{(\mathbf{1})}$.

In an analysis of heart failure with preserved ejection fraction (EF), type $2 \mathrm{DM}$ was associated with a significantly increased risk of developing adverse outcomes of heart failure ${ }^{(2)}$. To prevent the progression of heart failure in diabetic patients, a sensitive method of diagnosing the presence of diabetic cardiomyopathy is important ${ }^{(3)}$. However, clinical diagnostic surrogates to monitor myocardial disease progression in diabetic patients are not well established ${ }^{(4)}$.

Myocardial strain echocardiography can detect myocardial damage beyond that indicated by EF by assessing myocardial deformation in any direction ${ }^{(5)}$.

Assessment of LV systolic function may be carried out by either conventional echocardiogram or by speckle tracking strain imaging and several studies emerged over last few years reporting the use of speckle tracking strain imaging to detect subtle changes in the ventricles ${ }^{\left({ }^{(}\right)}$. Speckle-tracking echocardiography has recently emerged as a quantitative ultrasound technique for accurately evaluating myocardial function by analyzing the motion of speckles identified on routine 2-dimensional sonograms. It provides non-Doppler, angle-independent, and objective quantification of myocardial deformation and left ventricular systolic and diastolic dynamics. By tracking the displacement of the speckles during the cardiac cycle, strain and the strain rate can be rapidly measured offline after adequate image acquisition (7).

Speckle tracking imaging allows angleindependent evaluation of multidirectional LV strain in radial (RS), circumferential (CS) and longitudinal (LS) directions, and RV longitudinal strain ${ }^{(6)}$.

The study was aimed at early detection of subtle left ventricular systolic dysfunction by speckle tracking echocardiography in patients with Diabetes Mellitus with and without hypertension.

\section{PATIENTS AND METHODS}

This study included a total of 100 asymptomatic poorly controlled diabetic patients with or without hypertension and no structural heart disease and 25 age-matched apparently healthy control subjects attending at Cardiology department, Al-Azhar University Hospital in Damietta. Approval of the ethical committee and a written informed consent from all the subjects were obtained.

This study was conducted between August 2018 to July 2019.

The included subjects were divided into three groups; Group I (control) consisted of 25 apparently healthy subjects, Group II (diabetic normotensive group) consisted of 64 patients and Group III (diabetic hypertensive) consisted of 36 patients.

\section{Exclusion criteria:}

1) Patients with coronary artery disease.

2) ECG abnormalities. 
3) LV wall motion abnormalities on echocardiography.

4) Significant valvular heart disease.

5) Rhythm other than normal sinus rhythm.

6) $\mathrm{LVEF}<50 \%$.

1. Thorough Clinical Examination and History Taking: for assessment of Cardiac symptoms (palpitation, syncope, chest pain and exertional or resting dyspnea). A detailed medical history was obtained from all patients including cardiovascular risk factors, history of previous medication intake and history of other medical problem with special emphasis on antidiabetic and antihypertensive drugs. Body mass index (BMI) was calculated for all patients as follows: $\mathrm{BMI}=$ weight $/\left(\right.$ height in meter $^{2}$ )

2. Laboratory determination of HbA1c: all patients included in the study were subjected to this test to confirm poor control of diabetes before enrolling the patient in the study.

3. Resting Electrocardiogram: Twelve leads ECG for signs of ischemia as pathological $\mathrm{Q}$ wave $\mathrm{ST}-\mathrm{T}$ changes (normal, non-specific ECG changes).

4. Resting conventional Trans-Thoracic Echocardiography: Patients underwent transthoracic echocardiography at admission using $X$ 5-1 matrix array probe (frequency range 1.5-4.3 MHz) connected to Philips IE 33 machine (Philips healthcare, MA, USA).

5. Speckle Tracking Echocardiography (STE):

- Image acquisition: With simultaneous ECG recording, 2D echocardiography images were obtained from apical window (to get $\mathrm{AP} 4 \mathrm{C}, \mathrm{AP} 2 \mathrm{C}$ and $\mathrm{AP} 3 \mathrm{C}$ views). All images were obtained during breath hold and stored in cine-loop format from three or more consecutive beats. The frame rate of images was between 50 and 90 frames/s.

- Image Analysis: For each of the apical views (AP4C, AP2C and AP3C), 3 sampling points were placed manually at each side of the mitral annulus and at the apical endocardium. A region of interest (ROI) was then generated by the software to cover the entire thickness along the LV myocardium. The ROI was adjusted manually to provide optimal tracking.

The GLS values were estimated after the aortic valve closure had been identified visually, frame-byframe, in the AP3C view. When a segment had a great difference in segmental longtuidinal strain (SLS) compared with a similarly contracted neighbored segment, SLS was recalculated; if the problem could not be resolved, the segment was excluded.

SLS values were based on the American Society of Echocardiography's 17-segment LV model (8). Segmental LS was calculated as the percentage of lengthening or shortening of each segment and global longtuidinal stain (GLS) was calculated as the average of regional strains.

The myocardium of the LV in apical views was automatically divided into 6walls: infero-septumand lateral in AP4C view, anterior and inferior in AP2C view and antero-septum and inferolateralin AP3C view. Each wall is further divided into basal, midand apical levels ${ }^{(8)}$ as shown in (figure 1).

The results of all three planes were represented in a single bull's-eye summary as global peak longtuidinal strain (GPLS) along with segmental and global peak circunfrntial strain (GPCS) as shown in (figure 3).

The globalpeak circumferential strain (GPCS) was assessed from the short axis views at LV basal, mid and apical levels by a dedicated algorithm using a protocol similar to that of longitudinal strain analysis. In the short axis view, the myocardium of the LV was divided into 6 segments in both basal and mid-levels while the apical level was divided into 4 segments. The segmental cicumfrential strain (SCS) was calculated for each segment and the average of the all segmental values comprised the GPCS as shown in (figure.2).
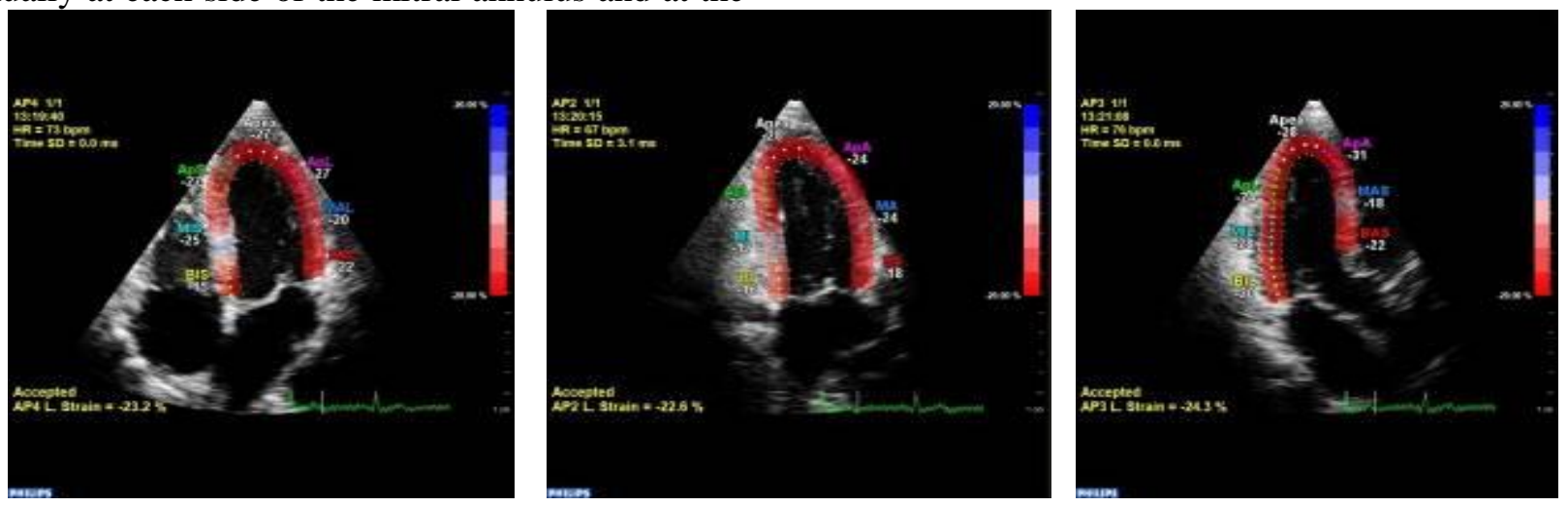

Figure (1): 2D longitudinal strain analysis of the LV as traced in the apical four-chamber, apical 2 chambers, apical 3 chamber views ${ }^{(8)}$. 

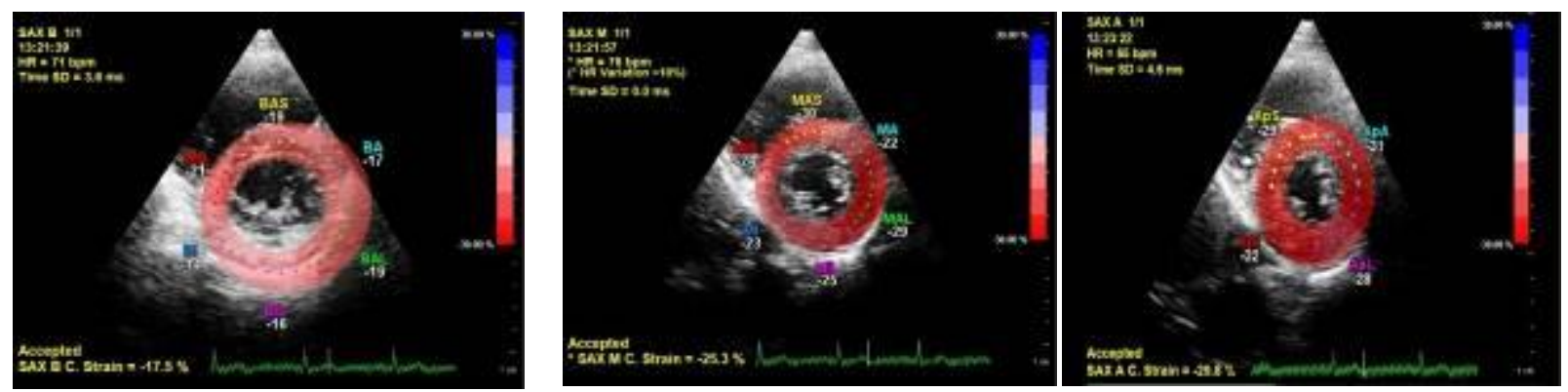

Figure (2): 2D circumferential strain analysis of the LV as traced in the basal, mid and apical short axis views ${ }^{(\mathbf{8})}$.
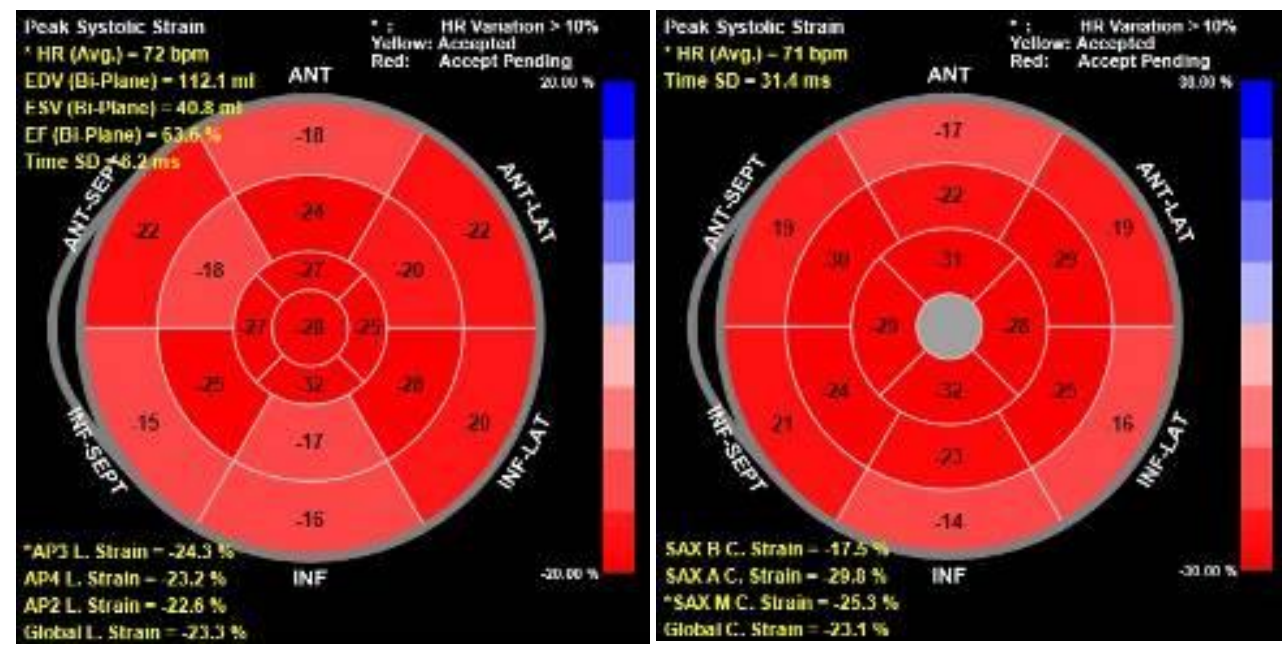

Figure (3): Bull's-eye summary of global peak longtuidinal strain values (GPLS) and global peak circumferential strain values (GPCS) as generated by the CMQ in a 17 -segment left ventricular model ${ }^{(8)}$.

\section{Data Collection, Statistical Analysis and Presentation:}

Data was collected and coded using Microsoft Office Excel Worksheet while statistical analysis was performed using "MedCalc Version 13.0.6" and statistical package for social sciences (SPSS) version 23.0 for windows. Both were used for graphical presentation.

In descriptive analysis:

- Categorical variables were expressed as frequency or percentages $(\%)$

- $\quad$ Continuous data were expressed as mean $\pm \mathrm{SD}$ (standard deviation) and range.

In inferential analysis we used the following:

- Chi-square $\left(\chi^{2}\right)$ and Fisher's exact tests to test for significance when comparing categorical variables.
- Unpaired sample t-test when comparing means of patients and controls.

- Paired sample t-test when comparing means of repeated measurements in patients.

- Probability (P-value)

- P-value $\leq 0.05$ was considered significant.

- P-value $\leq 0.001$ was considered highly significant.

- P-value $>0.05$ was considered insignificant.

- Pearson's correlation coefficient (r) test for correlating data and the results were interpreted as follows:

- $0.0=r:$ no correlation

- $0.0<\mathrm{r}<0.2$ : very weak (poor) correlation

- $0.2<\mathrm{r}<0.4$ : weak correlation

- $0.4<r<0.6$ : moderately strong correlation

- $0.6<\mathrm{r}<0.8$ : strong correlation

- $0.8<\mathrm{r}<1.0$ : very strong correlation

- $1.0=\mathrm{r}$ : perfect correlation.

- $\quad$ Student $t$ test (two-tailed) to test for significance when comparing continuous variables:

\section{RESULTS}

Table (1): Comparison between studied groups as regard to patient gender

\begin{tabular}{|c|c|c|c|c|c|c|c|c|c|}
\hline & \multicolumn{6}{|c|}{ Group } & \multirow{2}{*}{\multicolumn{2}{|c|}{$\begin{array}{c}\text { Total } \\
(\mathrm{n}=125)\end{array}$}} \\
\hline & & \multicolumn{2}{|c|}{ Control $(n=25)$} & \multicolumn{2}{|c|}{ Group II (DM(n=64) } & \multicolumn{2}{|c|}{ Group III (DM+HTN(n=36) } & & \\
\hline & & $\mathrm{n}$ & $\%$ & $\mathrm{~N}$ & $\%$ & $\mathrm{n}$ & $\%$ & $\mathrm{n}$ & $\%$ \\
\hline \multirow[t]{2}{*}{ Sex } & Male & 13 & $52.0 \%$ & 28 & $43.8 \%$ & 19 & $52.8 \%$ & 60 & $48.0 \%$ \\
\hline & Female & 12 & $48.0 \%$ & 36 & $56.3 \%$ & 17 & $47.2 \%$ & 65 & $52.0 \%$ \\
\hline \multicolumn{2}{|c|}{ Statistics } & \multicolumn{8}{|c|}{$\mathrm{st}=0}$. \\
\hline
\end{tabular}


The present study included 25 control subjects; 13 of them (52.0\%) were males and $12(48.0 \%)$ were females. In addition, it included 64 patients with diabetes mellitus (DM) without hypertension (HTN) (Group II); 28 of them (43.8\%) males and $36(56.3 \%)$ females. In addition, there were 36 patients with DM plus HTN (Group III); this groups included $19(52.8 \%)$ males and $17(47.2 \%)$ females. Thus, the total number of males were 60 representing $48.0 \%$ and total number of females were 65 representing 52\%. There was no significant difference between the groups.

Table (2): Comparison between groups II and III as regard to duration (years) of diabetes

\begin{tabular}{|c|c|c|c|c|c|c|}
\hline & Mean & S D & Min. & Max. & t & p \\
\hline Group II (DM) & 9.10 & 2.46 & 5.00 & 13.00 & \multirow{3}{*}{0.32} & \multirow{2}{*}{0.74} \\
\hline Group III (DM+HTN) & 9.27 & 2.53 & 5.00 & 14.00 & \\
\hline Total & 9.17 & 2.47 & 5.00 & 14.00 & & \\
\hline
\end{tabular}

In the present work, diabetes mellitus duration among studied patients ranged from 5 to 14 years and there was no significant difference between group II and III as regard to duration of DM ( $9.10 \pm 2.46$ vs $9.27 \pm 2.53$ years respectively).

Table (3): Comparison between studied groups as regard to BMI of studied subjects

\begin{tabular}{|c|c|c|c|c|c|c|}
\hline & Mean & S. D & Min. & Max. & $\mathrm{F}$ & $\mathrm{p}$ \\
\hline Control & 22.24 & 2.17 & 20.00 & 26.00 & \multirow{4}{*}{20.71} & \multirow{4}{*}{$<0.001 *$} \\
\hline Group II (DM) & 25.27 & 3.40 & 19.00 & 30.00 & & \\
\hline Group III & 27.39 & 2.99 & 22.00 & 32.00 & & \\
\hline Total & 25.27 & 3.53 & 19.00 & 32.00 & & \\
\hline \multirow{3}{*}{$\begin{array}{l}\text { Two groups } \\
\text { Comparisons }\end{array}$} & \multicolumn{4}{|c|}{ Control vs group II } & $\mathrm{t}=4.13$ & $<0.001 *$ \\
\hline & \multicolumn{4}{|c|}{ Control vs Group III } & $\mathrm{t}=7.36$ & $<0.001^{*}$ \\
\hline & \multicolumn{4}{|c|}{ Group II vs III } & $\mathrm{t}=3.13$ & 0.002* \\
\hline
\end{tabular}

Body mass index (BMI) among studied patients ranged from 19 to $32 \mathrm{~kg} / \mathrm{m}^{2}$ and there was statistically significant decrease of BMI in control group 22.24 \pm 2.17 , when compared to group II (25.27 \pm 3.40 ) or group III (27.39 \pm 2.99$)$. In addition, BMI was significantly decreased in group II when compared to group III $(25.27 \pm 3.40 \mathrm{vs}$ $27.39 \pm 2.99$, and $\mathrm{p}$ value $=0.002$ ).

Table (4): Comparison between studied groups as regard to blood pressure ( $\mathrm{mmHg}$ ):

\begin{tabular}{|c|c|c|c|c|c|c|c|}
\hline & & Mean & SD & Min. & Max. & $\mathrm{F}$ & $\mathrm{p}$ \\
\hline \multirow{4}{*}{ SBP } & Control & 114.20 & 11.52 & 95.00 & 135.00 & \multirow[t]{4}{*}{31.80} & \multirow{4}{*}{$<0.001 *$} \\
\hline & Group II & 115.31 & 13.03 & 90.00 & 130.00 & & \\
\hline & Group III & 134.17 & 10.99 & 110.00 & 150.00 & & \\
\hline & Total & 120.52 & 14.90 & 90.00 & 150.00 & & \\
\hline \multirow{3}{*}{\multicolumn{2}{|c|}{$\begin{array}{l}\text { Two groups } \\
\text { comparison }\end{array}$}} & \multicolumn{4}{|c|}{ Control vs group II } & $t=0.37$ & $0.71(\mathrm{~ns})$ \\
\hline & & \multicolumn{4}{|c|}{ Control vs Group III } & $t=6.84$ & $<0.001 *$ \\
\hline & & \multicolumn{4}{|c|}{ Group II vs III } & $t=7.33$ & $<0.001 *$ \\
\hline \multirow[t]{4}{*}{ DBP } & Control & 69.20 & 7.73 & 60.00 & 85.00 & \multirow[t]{4}{*}{12.38} & \multirow[t]{4}{*}{$<0.001 *$} \\
\hline & Group II & 71.33 & 7.83 & 60.00 & 85.00 & & \\
\hline & Group III & 78.89 & 10.01 & 65.00 & 95.00 & & \\
\hline & Total & 73.08 & 9.24 & 60.00 & 95.00 & & \\
\hline \multirow{3}{*}{\multicolumn{2}{|c|}{$\begin{array}{l}\text { Two groups } \\
\text { comparison }\end{array}$}} & \multicolumn{4}{|c|}{ Control vs group II } & $t=1.15$ & $0.25(\mathrm{~ns})$ \\
\hline & & \multicolumn{4}{|c|}{ Control vs Group III } & $t=4.06$ & $<0.001 *$ \\
\hline & & \multicolumn{4}{|c|}{ Group II vs III } & $t=4.18$ & $<0.001 *$ \\
\hline
\end{tabular}

Systolic blood pressure among studied populations ranged from 90 to $150 \mathrm{mmHg}$, while diastolic blood pressure ranged from 60 to $95 \mathrm{mmHg}$; and there was significant increase of both systolic and diastolic blood pressure in group III when compared to either group II or control group. However, the difference between control group and group II was statistically non-significant. 
Table (5): Comparison between studied groups as regard to glycated hemoglobin (\%):

\begin{tabular}{|c|c|c|c|c|c|c|}
\hline $\mathrm{HbA1c}$ & Mean & S. D & Min. & Max. & $\mathrm{F}$ & $\mathrm{p}$ \\
\hline Control & 5.62 & 0.42 & 5.00 & 6.20 & \multirow{4}{*}{106.81} & \multirow{4}{*}{$<0.001 *$} \\
\hline Group II (DM) & 9.59 & 1.28 & 7.50 & 12.50 & & \\
\hline Group III & 8.99 & 1.28 & 7.50 & 11.50 & & \\
\hline Total & 8.62 & 1.92 & 5.00 & 12.50 & & \\
\hline Two groups & \multicolumn{4}{|c|}{ Control vs group II } & $t=15.11$ & $<0.001 *$ \\
\hline Comparisons & \multicolumn{4}{|c|}{ Control vs Group III } & $t=12.61$ & $<0.001 *$ \\
\hline & \multicolumn{4}{|c|}{ Group II vs III } & $\mathrm{t}=\mathbf{2 . 2 7}$ & $0.025^{*}$ \\
\hline
\end{tabular}

In the present work, glycated hemoglobin ranged from 5 to 12.50 and there was significant decrease in control group $(5.62 \pm 0.42)$ when compared to group II $(9.59 \pm 1.28)$ or group III $(8.99 \pm 1.28)$. In addition, there was significant increase of glycated hemoglobin in group II when compared to group III.

Table (6): Comparison between groups II and III as regard to anti-diabetic drug therapy

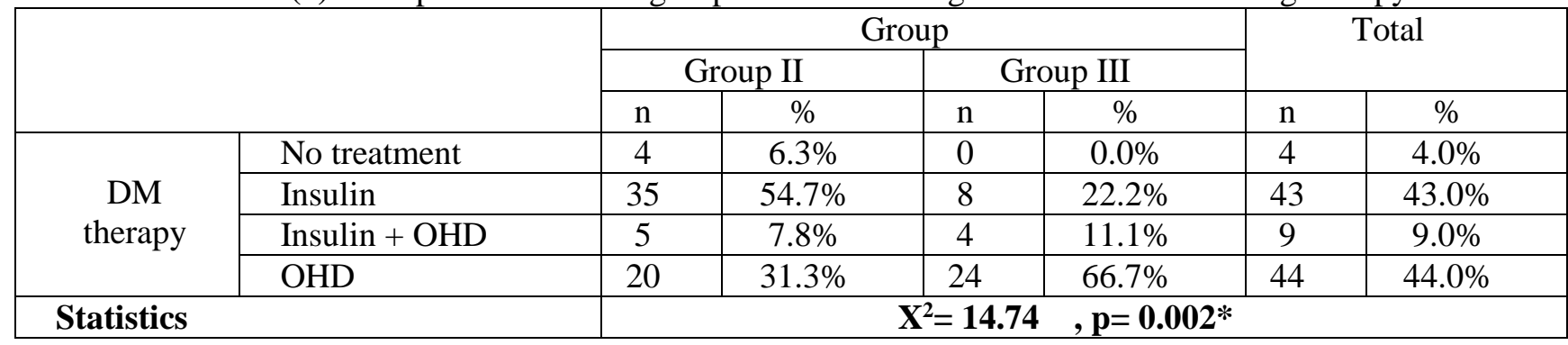

As regard to anti-diabetic therapy, there were 4 patients (4.0\%) who not take anti-diabetic drugs, 43 patients (43\%) used insulin, 9 patients $(9.0 \%)$ were on insulin plus oral hypoglycemic drugs (OHD) and finally 44 patients (44.0\%) were on OHD, and there was significant difference between groups II and III as regard to anti-diabetic drugs, where there was significant increase of percentage of patients on insulin and no treatment and significant decreases of insulin plus OHD and OHD in group II when compared to group III $(6.3 \%, 54.7 \%, 7.8 \%$ and $31.3 \%$ vs $0.0 \%, 22.2 \%, 11.1 \%$ and $66.7 \%$ respectively).

Table (7): Correlation between longitudinal strain and other variables

\begin{tabular}{|l|c|c|c|c|c|c|}
\hline & \multicolumn{2}{|c|}{ Ls_4ch } & \multicolumn{2}{c|}{ Ls_2ch } & \multicolumn{2}{c|}{ Ls_3ch } \\
\cline { 2 - 7 } & $\mathbf{r}$ & $\mathbf{p}$ & $\mathbf{r}$ & $\mathbf{p}$ & $\mathbf{r}$ & $\mathbf{p}$ \\
\hline DM duration & $\mathbf{0 . 3 3 9} * *$ & $\mathbf{0 . 0 0 1}$ & $\mathbf{0 . 2 1 5} *$ & $\mathbf{0 . 0 3 1}$ & 0.068 & 0.500 \\
\hline BMI & $\mathbf{0 . 3 1 2 * *}$ & $\mathbf{0 . 0 0 2}$ & $\mathbf{0 . 4 6 0} * *$ & $\mathbf{0 . 0 0 1}$ & 0.183 & 0.068 \\
\hline SBP & 0.023 & 0.824 & 0.128 & 0.205 & 0.066 & 0.513 \\
\hline DBP & 0.095 & 0.349 & 0.127 & 0.208 & 0.088 & 0.384 \\
\hline HbA1c & -0.049 & 0.625 & -0.080 & 0.430 & -0.063 & 0.536 \\
\hline LVESD & -0.045 & 0.654 & 0.041 & 0.686 & -0.030 & 0.770 \\
\hline LVEDD & -0.074 & 0.463 & 0.138 & 0.170 & 0.037 & 0.716 \\
\hline LVESV & 0.064 & 0.529 & -0.025 & 0.807 & -0.107 & 0.290 \\
\hline LVED & 0.017 & 0.868 & 0.015 & 0.884 & -0.057 & 0.570 \\
\hline EF\% & -0.028 & 0.779 & 0.090 & 0.371 & 0.111 & 0.271 \\
\hline
\end{tabular}

As regard to correlation between longitudinal strain and studied variables, each of DM duration and BMI correlates with each of LS-4ch and LS-2ch. 
Table (8): Correlation between circumferential strain and other variables among studied populations

\begin{tabular}{|l|c|c|c|c|c|c|}
\hline \multirow{2}{*}{} & \multicolumn{2}{|c|}{ CS-MV } & \multicolumn{2}{c|}{ CS-PM } & \multicolumn{2}{c|}{ CS-AP } \\
\cline { 2 - 6 } & $\boldsymbol{r}$ & $\boldsymbol{p}$ & $\boldsymbol{r}$ & $\boldsymbol{p}$ & $\boldsymbol{r}$ & $\boldsymbol{p}$ \\
\hline DM duration & .149 & .138 & .163 & .105 &. $\mathbf{2 0 9}$ & $\mathbf{. 0 3 7}$ \\
\hline BMI & .018 & .862 & .129 & .200 & .187 & .063 \\
\hline SBP & .025 & .807 & -.027 & .791 & .048 & .638 \\
\hline DBP & .059 & .557 & .029 & .776 & .104 & .302 \\
\hline HbA1c & -.168 & .095 & -.116 & .252 & .091 & .369 \\
\hline LVESD & .091 & .369 & .053 & .601 & -.098 & .332 \\
\hline LVEDD & .133 & .186 & -.044 & .664 & -.081 & .421 \\
\hline LVESV & -.083 & .414 & .024 & .814 & -.034 & .738 \\
\hline LVED & -.090 & .374 & -.072 & .475 & -.060 & .552 \\
\hline EF\% & .010 & .922 & -.123 & .224 & .064 & .524 \\
\hline
\end{tabular}

In diabetic groups, circumferential strain of AP was correlated with DM duration. However, no other correlation were found.

Table (9): Correlation between left ventricle GLS and GCS with other variables

\begin{tabular}{|l|c|c|c|c|}
\hline \multirow{2}{*}{} & \multicolumn{2}{|c|}{ LV-GLS } & \multicolumn{2}{c|}{ LV-GCS } \\
\cline { 2 - 5 } & $\boldsymbol{r}$ & $\boldsymbol{p}$ & $\boldsymbol{r}$ & $\boldsymbol{p}$ \\
\hline DM duration & $\mathbf{. 2 5 1 ^ { * }}$ & $\mathbf{. 0 1 2}$ & $\mathbf{. 2 5 8 ^ { * * }}$ & $\mathbf{. 0 1 0}$ \\
\hline BMI & $\mathbf{. 2 3 1 ^ { * }}$ & $\mathbf{. 0 2 1}$ & .146 & .146 \\
\hline SBP & -.113 & .265 & -.107 & .287 \\
\hline DBP & .162 & .106 & .038 & .704 \\
\hline HbA1c & .167 & .097 & .118 & .243 \\
\hline LVESD & .071 & .484 & .093 & .360 \\
\hline LVEDD & .065 & .519 & .043 & .671 \\
\hline LVESV & -.033 & .742 & -.020 & .845 \\
\hline LVED & -.117 & .246 & -.133 & .186 \\
\hline EF\% & -.031 & .759 & -.084 & .407 \\
\hline
\end{tabular}

Among diabetic groups, LV-GLS, was correlated with each of DM duration and BMI; while LV-GCS was significantly correlated with DM duration. Otherwise no significant correlation was found for other variables with global strain.

\section{DISCUSSION}

Diabetes mellitus is often associated with coronary risk factors, resulting in significant cardiac morbidity and mortality. Early detection of diabetic heart disease is of paramount importance, because timely life-style modifications and medical interventions could prevent or delay the subsequent development of heart failure which is considered one of major burdens for health insurance costs ${ }^{(9)}$.

As diabetes may cause impairment of left ventricular functions, a condition is known as "Diabetic cardiomyopathy" that was first described in 1972, 4 cases with both diabetes and heart failure but neither arterial hypertension nor $\mathrm{CAD}$ and by anatomical dissection of the heart it revealed LV hypertrophy and fibrosis without evidence of coronary artery atheroma ${ }^{(\mathbf{1 0})}$.
So, attention of researchers had been paid for early detection of LV dysfunction in patients with diabetes for improvement of morbidity and mortality. Diabetic patients with normal left ventricular ejection fraction (LVEF) are frequently associated with diastolic dysfunction. However, neither diastolic function nor LVEF is known not to be a sensitive marker for the detection of subclinical LV systolic dysfunction as diastolic function is widely affected by other diseases and ageing process which could make it less sensitive method of detection of early LV dysfunction due to diabetic cardiomyopathy ${ }^{(\mathbf{1 0})}$.

Early manifestation of diabetic LV systolic dysfunction can be detected longitudinally, because subendocardial fibers, which are prone to myocardial ischemia, have a longitudinal trajectory.

The presence of impaired longitudinal function in diabetic and/or hypertensive patients has been reported when using tissue Doppler imaging. However, tissue Doppler imaging has its own limitations including angle dependency and the 1D nature of its measurement as studied by Pellerin et al. ${ }^{(11)}$.

In contrast, Two-dimensional Speckle-tracking echocardiography (2D STE) has the advantage that it 
allows the measurement of all principal LV strains in an angle independent manner, thus eliminating the major limitation of tissue Doppler imaging similar to previous tissue Doppler studies.

The objectives of this study were to early detect subtle LV systolic dysfunction in patients with diabetes using 2D STE to assess both longitudinal strains (LS-4ch, LS-2ch, LS-3ch), Global longitudinal strain (GLS), and circumferential strains (CS-MV,CS-PM,CS-AP) , Global circumferential strain (GCS).

In the present study, 100 consecutive patients with poorly controlled diabetes (proved with high levels of HbAlc >7) were included in the study in addition to apparently healthy age and sex matched control divided into 3 groups:

1. Apparently healthy age and sex matched patients in control group (group I) which comprised 25 patients.

2. Diabetic normotensive patients (group II) which comprises 64 patients.

3. Diabetic hypertensive patients (group III) which comprises 36 patients.

Fifty two percent of the studied patients were females and forty-eight were males.

In the present study patient age ranged from 42 to 61 years and there was no difference between control and case groups.

Diabetes mellitus duration among studied patients ranged from 5 to 14 years and there was no significant statistical difference between group II and III as regard to duration of $\mathrm{DM}(9.10 \pm 2.46 \mathrm{Vs} 9.27 \pm 2.53$ years respectively).

Systolic blood pressure among studied populations ranged from 90 to $150 \mathrm{mmHg}$, while diastolic blood pressure ranged from 60 to $95 \mathrm{mmHg}$; and there was significant increase of both systolic and diastolic blood pressure in group III when compared to either group II or control group.

However, there was no correlation between systolic or diastolic blood pressure with either global longitudinal or circumferential strains in contrary to Wolf et al. (12), who found that HT causes the development of subendocardial myocardial damage and results in LS abnormality. In their clinical investigation, Wolf et al. ${ }^{(12)}$ reported that HT without ventricular hypertrophy caused LS impairment. However, there was no significant difference in the strain parameters of groups II and III in the present study. A possible explanation for the lack of a significant effect of HT on strain abnormality is that it may be related to the high prevalence of patients taking antihypertensive medications in our study.

Most of patients had been on antidiabetic (oral hypoglycemic, insulin or both) and antihypertensive (beta-blocker, calcium channel blocker, ACE-I and ARBS ) before the study.
Body mass index (BMI) among studied patients ranged from 19 to $32 \mathrm{~kg} / \mathrm{m} 2$ and there was statistically significant decrease of BMI in control group (22.24 \pm 2.17$)$, when compared to group II $(25.27 \pm 3.40)$ or group III (27.39 \pm 2.99$)$. In addition, BMI was significantly decreased in group II when compared to group III $(25.27 \pm 3.40 \mathrm{Vs} 27.39 \pm 2.99$, and $\mathrm{p}$ value $=0.002)$.

BMI was significantly correlated with LS-4ch, LS2ch, CS-AP and GLS but not GCS. These results are consistent with Cozzolino et al. (1), who reported progressive longitudinal systolic dysfunction associated with obesity.

Regarding 2D conventional echocardiographic parameters, mean LV-EF in groups II, III was (64.98 \pm 4.08$),(65.5 \pm 4.13)$ respectively, and in control group $(65.16 \pm 3.83)$ with no statistical difference between the groups. As regard LVEDV, in groups II, III mean LV EDV was ( $103.75 \pm 14.08)$ ,(104.01 \pm 16.01$)$ respectively while in control group was $(108.08 \pm 15.73)$, it seems to be mild decreased in case groups but without statistical significance $(\mathrm{P}=0.45)$. As regard LV- ESV, in groups II, III mean LVESV was (36.21 \pm 5.89$)$, (35.38 \pm 6.54$)$ respectively while in control group was $(37.28 \pm 6.70)$ with no statistically significance between the groups $(\mathrm{P}=0.51)$.

Regarding 2D speckle tracking variables of global function, in our study there was a significant difference as regard LV-GLS and LV-GCS between case and control groups. Global values of LV were significantly lower in both groups II,III than in the control subjects with $p<0.001$ as regard both GLS and GCS, indicating that patients with diabetes (groups II,III), have a decrease in global strain of the LV in contrast to healthy subjects, whereas LV function measured by conventional echocardiographic techniques were normal. This indicates that diabetes may induce early LV dysfunction not detected by conventional 2D echocardiography measurements and this finding is consistent with Wang et al. ${ }^{(13)}$.

Global LS in control subject was $(-22.88 \pm 2.24)$. This were used to establish abnormal cut-off value of global LS. This was calculated as the value of the mean 2 2SD. Using the cut-off value of (-18.4) we observed that global and regional LSs were significantly reduced in diabetic patients with $56 \%$ of the patients showing abnormal LS values compared with the normal range obtained from our control group of age-matched subjects.

CS at the base- and the mid-LV levels did not differ between groups. However, CS at the apical level was significantly lower in diabetic patients, resulting in a significant reduction in global CS this finding is consistent with Gnakamene et al. ${ }^{(14)}$.

The present study showed that diabetic duration was the only independent predictor for the reduction in LS. This highlights the relationship between long-term 
hyper-glycaemia and the impairment of LS this finding is consistent with Lachin et al. ${ }^{(15)}$.

No significant correlation was noted between the reduction of global values of Longitudinal, circumferential strain and glycosylated hemoglobin (HbA1c), with ( $\mathrm{P}=.097$ and .243 respectively) in contrary to Zhao et al. ${ }^{(16)}$ who suggested a graded positive association of $\mathrm{HbAlc}$ with the risk of $\mathrm{HF}$ among both African American and white patients with diabetes this may be attributed to the type of the study as Zhao et al. ${ }^{(\mathbf{1 6})}$ followed their patient for up to 6 years while our study is a retrospective one.

\section{CONCLUSION}

It could be concluded that assessment of left ventricular (LV) systolic function in patients with diabetes mellitus by speckle tracking echocardiographic modalities can detect subtle LV dysfunction earlier than conventional echocardiograohic measures.

\section{RECOMMENDATIONS}

The physicians should be aware the condition when examining a patient with long standing diabetes mellitus as it may be an important risk factor of progressing LV dysfunction and heart failure even in absence of structural heart disease.

Speckle tracking echocardiographic modalities have the upper hand in assessment of subtle LV dysfunction not detected by conventional methods. As they can detect LV dysfunction earlier than conventional methods, they may be considered the best non-invasive technique in assessment of LV dysfunction in patients with diabetes and therefore early detection of LV dysfunction which help physicians in improvement of mortality and morbidity.

\section{REFERENCES}

1. Cozzolino M, Galassi A, Pivari F et al. (2017): The cardiovascular burden in end-stage renal disease. In Expanded Hemodialysis, 12: 44-57.

2. Okuyama R, Ishii J, Takahashi H et al. (2017): Combination of high-sensitivity troponin I and N-terminal pro-B-type natriuretic peptide predicts future hospital admission for heart failure in high-risk hypertensive patients with preserved left ventricular ejection fraction. Heart and Vessels, 32(7): 880-892.

3. Tate M, Deo M, Cao AH et al. (2017): Insulin replacement limits progression of diabetic cardiomyopathy in the low-dose streptozotocin-induced diabetic rat. Diabetes and Vascular Disease Research, Diab Vasc Dis Res., 14(5):423-433.
4. Ho CY, McMurray JJ, Cirino AL et al. (2017): Valsartan for attenuating disease evolution in early sarcomeric hypertrophic cardiomyopathy: The design of the Valsartan for Attenuating Disease Evolution in Early Sarcomeric Hypertrophic Cardiomyopathy (VANISH) trial. American Heart Journal, 187: 145-155.

5. Spartera M, Damascelli A, Mozes F et al. (2017): Threedimensional speckle tracking longitudinal strain is related to myocardial fibrosis determined by late-gadolinium enhancement. The International Journal of Cardiovascular Imaging, 9: 1-10.

6. Furian T, Aguiar C, Prado K et al. (2012): Ventricular dysfunction and dilation in severe sepsis and septic shock: relation to endothelial function and mortality. J Crit Care, 27: 319-25.

7. Mondillo S, Cameli M, Solari M et al. (2016): Echocardiographic assessment of left ventricular systolic function: from ejection fraction to torsion. Heart Failure Reviews, 21: 77-94.

8. Lang RM, Badano LP, Mor-Avi V et al. (2015): Recommendations for cardiac chamber quantification by echocardiography in adults: an update from the American Society of Echocardiography and the European Association of Cardiovascular Imaging. J Am Soc Echocardiogr., 28(1):1-39.

9. Frederix I (2018): Cardiac telerehabilitation: a novel, costefficient care delivery strategy in secondary prevention for ischemic heart disease? University of Antwerp. https://www.researchgate.net/publication/281109634_

10. Voulgari C, Papadogiannis D and Tentolouris N (2010): Diabetic cardiomyopathy: from the pathophysiology of the cardiac myocytes to current diagnosis and management strategies. Vascular Health and Risk Management, 6: 883-890.

11. Pellerin D, Sharma R, Elliott P et al. (2003): Tissue Doppler, strain, and strain rate echocardiography for the assessment of left and right systolic ventricular function. Heart, 89: 9-17.

12. Wolf M, Lucina SB, Brüler BC et al. (2018): Assessment of longitudinal systolic function using tissue motion annular displacement in healthy dogs. Journal of Veterinary Cardiology, 20: 175-185.

13. Wang Q, Hu L, Sun A et al. (2018): Assessment of global and regional strain left ventricular in patients with preserved ejection fraction after Fontan operation using a tissue tracking technique. The International Journal of Cardiovascular Imaging, 11: 1-8.

14. Gnakamene JB, Safar ME, Levy BI et al. (2018): Left Ventricular Torsion Associated With Aortic Stiffness in Hypertension. Journal of the American Heart Association, 7: e007427.

15. Lachin JM, Orchard TJ and Nathan DM (2014): Update on cardiovascular outcomes at 30 years of the diabetes control and complications trial/ epidemiology of diabetes interventions and complications study. Diabetes Care, 37:3943.

16.Zhao Q, Zhang Y, Niu Y et al. (2018): Comparison of angiotensin-converting enzyme inhibitors and angiotensin II receptor blockers on cardiovascular outcomes in hypertensive patients with type 2 diabetes mellitus: A PRISMA-compliant systematic review and meta-analysis. Medicine, 97(15):e0256. 